\title{
Nível de atividade física, condições de saúde e características sócio-demográficas de mulheres idosas brasileiras
}

\author{
G Z Mazo \\ $\mathrm{J} \mathrm{Mota}^{2}$ \\ LHT Gonçalves ${ }^{3}$ \\ MG Matos ${ }^{4}$ \\ https://doi.org/10.5628/rpcd.05.02.202
}

\section{RESUMO}

O objetivo deste estudo foi verificar o nível de atividade física e a sua relação com as características sócio-demográficas e as condições de saúde de mulheres idosas. A amostra foi composta por 198 mulheres idosas com 65 anos ou mais de idade $(M=73.6$ anos; $\mathrm{DP}=5.9$ ), que participam de Grupos de Convivência de Idosos na cidade de Florianópolis, SC, Brasil. A recolha de dados se deu em forma de entrevista individual aplicando-se: $i$ ) Formulário de identificação com dados das variáveis sócio-demográficas e de condições de saúde; ii) Questionário Internacional de Atividade Física (IPAQ), versão 8, forma longa e semana normal; iii) Questionário de Critério de Classificação Econômica do Brasil. Os dados foram analisados através da estatística descritiva e de testes não-paramétricos. Entre os resultados observou-se que $66 \%$ das idosas classifica-se no nível de atividade física mais ativo. Entre as atividades físicas exercidas, destacam-se as atividades domésticas $(40 \%)$ e de lazer (35\%). Verificou-se que há diferença significativa $(\mathrm{p}<0.05)$ entre os níveis de atividade física (menos e mais ativos) e as variáveis sócio-demográficas e de condições de saúde. Conclui-se que há a necessidade ainda de reforçar a intervenção nesta realidade, incentivando as idosas que são menos ativas a tornarem-se mais ativas, e as mais ativas a manterem-se nesta condição.

Palavras-chave: atividade física, idosas, características sóciodemográficas, condições de saúde.

\author{
${ }^{1}$ Universidade do Estado de Santa Catarina, Centro de Edu- \\ cação Física, Fisioterapia e Desportos, Florianópolis, Brasil \\ ${ }^{2}$ Universidade do Porto, Faculdade de Ciências \\ do Desporto e Educação Física, Portugal \\ ${ }^{3}$ Universidade Federal de Santa Catarina \\ Faculdade de Enfermagem, Florianópolis, Brasil \\ ${ }^{4}$ Universidade Técnica de Lisboa \\ Faculdade de Motricidade Humana, Portugal
}

\section{ABSTRACT}

Physical activity levels, health conditions and socio-demographic characteristics of Brazilian elderly women

The aim of this study was to verify the level of physical activity and the relationship between socio-demographic characteristics and health conditions of elderly women. Sample size comprises 198 elderly women aged 65 years and older $(M=73.6$ years; $D P=5.9)$, that participated in a special program in Florianópolis city, SC, Brazil. Data was collected by individual interview, and the information obtained relates to: 1) Identification form with socio-demographic and health conditions data; 2) International Physical Activity Questionnaire (IPAQ), version 8, extended form and normal week; 3) Questionnaire of Economic Classification Criteria of Brazil. Data was analyzed through the descriptive statistics and non-parametric tests. Results showed that $66 \%$ of the elderly women were classified in the most active level of physical activity, and the most practiced physical activities, according to the research, were housework (40\%) and leisure activities (35\%). It was verified that there is a significant difference $(p<0.05)$ between physical activity levels (more and less active) and the socio-demographic and health condition variables. Finally, it can be concluded that there is still a necessity to reinforce the intervention in this reality, stimulating the less active elderly to become more active, and the most active ones to remain in this condition.

Key Words: physical activity, elderly women, socio-demographic characteristics, health conditions. 


\section{INTRODUÇÃO}

O envelhecimento populacional nos países em desenvolvimento está a ocorrer num curto período de tempo. O Brasil será, em 2025, o sexto país em números absolutos, com mais de 30 milhões de idosos (17). O crescimento rápido da população idosa acarretará um grande impacto na economia do país e agravamento dos problemas nos setores sócio-econômicos e de saúde (26). Além disto, o processo de envelhecimento é acompanhado, muitas vezes, por um estilo de vida inativo, que favorece as incapacidades e dependência.

Neste contexto, torna-se importante conhecer as características sócio-demográficas, as condições de saúde e o nível de atividade física, pois estes aspectos da vida dos idosos podem tornar mais precoces ou mais tardias as alterações próprias do envelhecimento associadas à prevalência de doenças crônicodegenerativas. A partir do conhecimento desta realidade, podem ser investidos recursos econômicos e sociais em áreas necessárias que venham a manter, por mais tempo, a capacidade funcional dos idosos, como a implantação e/ou implementação de programas de atividade física.

Nos últimos anos, surgiu um aumento de programas de atividade física como meio de promoção de saúde dos idosos, pois foi estabelecido o papel da atividade física regular no processo de envelhecimento saudável, os efeitos fisiológicos, psicológicos, sociais e os benefícios de um estilo de vida fisicamente ativo ao longo do período de vida (39). Um consenso entre os investigadores reside no objetivo fundamental da prática de atividade física para os idosos, que é a preservação ou a restauração de uma qualidade de vida aceitável $(1,19,32)$.

O Brasil, dispõe de poucos dados sobre a prevalência da atividade física da sua população $(7,10,16)$, bem como da associação da inatividade física com as variáveis sócio-demográficas (idade, estado civil, etnia, ocupação, nível econômico entre outros) e as condições de saúde (presença ou não de doenças, tipo de doenças, percepção de saúde entre outras) dos idosos, principalmente utilizando as mesmas medidas de avaliação que possam subsidiar comparações nacionais e internacionais e indicadores de possíveis intervenções, em termos de saúde pública. Assim, temos como objetivo geral deste estudo veri- ficar os níveis de atividade física e a sua relação com as características sócio-demográficas e as condições de saúde de mulheres idosas. Como objetivos específicos, temos: (i) Verificar o nível de atividade física das idosas; e (ii) Identificar as características sóciodemográficas e as condições de saúde das idosas.

\section{MATERIAL E MÉTODOS \\ Amostra}

A amostra, do tipo probabilística, com a técnica de seleção aleatória estratificada, é composta por 198 mulheres idosas, de diferentes estratos etários (65 a 69 anos $=57 ; 70$ a 74 anos $=58 ; 75$ a 79 anos $=$ 48; 80 e mais anos $=35$ ), que participaram em 29 Grupos de Convivência para Idosos, distribuídos por 20 bairros e nos 12 Distritos da cidade de Florianópolis, SC, Brasil.

\section{Instrumentos}

Os instrumentos de recolha de dados, aplicados em forma de entrevista individual, foram: i) Formulário com os dados de identificação, sócio-demográficos e com as condições de saúde das idosas; ii) Questionário de Critério de Classificação Econômica do Brasil (6); iii) Questionário Internacional de Atividade Física (IPAQ), versão 8, forma longa e semana normal.

\section{Recolha de dados}

Os dados foram recolhidos pela pesquisadora e entrevistadoras, previamente treinadas. A aplicação da entrevista ocorreu, geralmente, no próprio espaço físico do grupo ou numa sala anexa a este.

Anteriormente a aplicação da entrevista, foi exposto às idosas selecionadas, o objetivo, a importância da pesquisa, o sigilo de identificação, o modo de aplicação e o destino dos dados obtidos. Uma vez que as idosas concordaram verbalmente em participar na pesquisa, foi aplicada a entrevista e, no final desta, assinada a formalização do convite para a participação na pesquisa em duas vias, ficando uma via na posse das idosas e outra na da pesquisadora.

\section{Tratamento estatístico}

Para a interpretação do nível de atividade física das idosas através do IPAQ foi utilizado o critério baseado em recomendações atuais de limiares de atividades físicas que resultam em benefícios para a saúde, 
classificando como "ativos" os indivíduos que praticam, pelo menos, 150 minutos por semana (min/sem) de atividade física, no mínimo moderada $(1,21,28)$. A partir deste critério, a amostra deste estudo foi dividida em dois níveis: menos ativas $(<150 \mathrm{~min} / \mathrm{sem})$ e mais ativas $(\geq 150 \mathrm{~min} / \mathrm{sem})$. Os dados foram analisados através da estatística descritiva e para a análise entre as variáveis categóricas foi utilizado o Teste do Qui-Quadrado e, quando necessário, foi adotado o teste Exato de Fisher. Adotou-se um nível de significância de 5\%.

\section{RESULTADOS}

Na Tabela 1, apresentam-se os cinco domínios do Questionário Internacional de Atividade Física IPAQ (no trabalho, transporte, atividades domésticas, lazer e no tempo gasto sentado) - com as suas diferentes atividades físicas e intensidades (vigorosa, moderada e leve).

Tabela 1. Número $(n]$, percentagem [\%], média $[\bar{X}]$ e desvios padrão (DP] da amostra, relativos aos minutos por semana (min/sem) e minutos por dia (min/dia), nos domínios do IPAQ. Florianópolis, SC, Brasil.

\begin{tabular}{|c|c|c|c|c|}
\hline Domínios do IPAD & $\mathrm{n}$ & $\%$ & $\begin{array}{c}\bar{X} \\
{[\mathrm{~min} / \mathrm{sem} ; \mathrm{min} / \mathrm{dia})}\end{array}$ & $\begin{array}{c}\text { DP } \\
{[\mathrm{min} / \mathrm{sem} ; \mathrm{min} / \mathrm{dia})}\end{array}$ \\
\hline \multicolumn{5}{|l|}{ AF no Trabalho } \\
\hline Trabalho moderado & $?$ & 3.5 & $530 \mathrm{~min} / \mathrm{sem}$ & $635 \mathrm{~min} / \mathrm{sem}$ \\
\hline Caminhada no trabalho moderada & 5 & 2.5 & $206 \mathrm{~min} / \mathrm{sem}$ & $200 \mathrm{~min} / \mathrm{sem}$ \\
\hline Caminhada no trabalho leve & 1 & 0.5 & $150 \mathrm{~min} / \mathrm{sem}$ & $\cdot$ \\
\hline \multicolumn{5}{|l|}{ AF no Transporte } \\
\hline Bicicleta moderada & 1 & 0.5 & $40 \mathrm{~min} / \mathrm{sem}$ & $\cdot$ \\
\hline Caminhada vigorosa & 2 & 1.0 & $65 \mathrm{~min} / \mathrm{sem}$ & $35 \mathrm{~min} / \mathrm{sem}$ \\
\hline Caminhada moderada & 78 & 39.4 & $114 \mathrm{~min} / \mathrm{sem}$ & $115 \mathrm{~min} / \mathrm{sem}$ \\
\hline Caminhada leve & 72 & 36.4 & $141 \mathrm{~min} / \mathrm{sem}$ & $351 \mathrm{~min} / \mathrm{sem}$ \\
\hline \multicolumn{5}{|l|}{ AF Doméstica } \\
\hline Ao redor da habitação vigorosa & 17 & 8.6 & $125 \mathrm{~min} / \mathrm{sem}$ & $208 \mathrm{~min} / \mathrm{sem}$ \\
\hline Ao redor da habitação moderada & 73 & 36.9 & $107 \mathrm{~min} / \mathrm{sem}$ & $116 \mathrm{~min} / \mathrm{sem}$ \\
\hline Dentro da habitação moderada & 121 & 61.1 & $230 \mathrm{~min} / \mathrm{sem}$ & $205 \mathrm{~min} / \mathrm{sem}$ \\
\hline \multicolumn{5}{|l|}{ AF Lazer } \\
\hline Caminhada vigorosa & 2 & 1.0 & $180 \mathrm{~min} / \mathrm{sem}$ & $\cdot$ \\
\hline Caminhada moderada & 60 & 30.3 & $239 \mathrm{~min} / \mathrm{sem}$ & $178 \mathrm{~min} / \mathrm{sem}$ \\
\hline Caminhada leve & 29 & 14.6 & $170 \mathrm{~min} / \mathrm{sem}$ & $181 \mathrm{~min} / \mathrm{sem}$ \\
\hline Lazer moderado & 104 & 52.5 & $112 \mathrm{~min} / \mathrm{sem}$ & $95 \mathrm{~min} / \mathrm{sem}$ \\
\hline \multicolumn{5}{|l|}{ Tempo Sentado } \\
\hline No transporte durante a semana & 153 & 77.3 & $176 \mathrm{~min} / \mathrm{sem}$ & $178 \mathrm{~min} / \mathrm{sem}$ \\
\hline Durante um dia da semana & 198 & 100.0 & $421 \mathrm{~min} / \mathrm{dia}$ & $140 \mathrm{~min} / \mathrm{dia}$ \\
\hline Durante um dia do Fim-de-semana & 198 & 100.0 & $472 \mathrm{~min} / \mathrm{dia}$ & $152 \mathrm{~min} / \mathrm{dia}$ \\
\hline
\end{tabular}

O domínio AF no trabalho inclui as atividades que as idosas executam no seu trabalho remunerado ou voluntário. Neste estudo, $24.7 \%$ (49) das idosas realizam trabalho remunerado e voluntário, sendo que $85.7 \%$ (42) desenvolvem atividades leves, do tipo fazer renda, pintura, artesanato e passar roupa a ferro, entre outras, enquanto $14.3 \%$ (7) realizam atividades moderadas. No IPAQ não é considerado o trabalho leve. Assim, das 198 idosas deste estudo, apenas $3.5 \%$ (7) fazem trabalho com intensidade moderada, em média de $530 \mathrm{~min} / \mathrm{sem}$. A caminhada, durante o trabalho, com intensidade vigorosa não é realizada, mas a moderada acontece em $2.5 \%$ das idosas, em média de $206 \mathrm{~min} / \mathrm{sem}$, e a leve é realizada por uma 
idosa $(0.5 \%)$, em média de $150 \mathrm{~min} / \mathrm{sem}$.

No domínio AF no transporte, que se relaciona com a deslocação de um lugar para o outro, apenas uma idosa $(0.5 \%)$ se desloca de bicicleta, com intensidade moderada, em média de $40 \mathrm{~min} / \mathrm{sem}$. A deslocação através da caminhada é a mais executada pelas idosas, sendo que $39.4 \%$ o fazem com intensidade moderada $(\overline{\mathrm{X}}=114 \mathrm{~min} / \mathrm{sem}), 36.4 \%$, com leve $(\bar{X}=141 \mathrm{~min} / \mathrm{sem})$ e $1.0 \%$, com vigorosa $(\overline{\mathrm{X}}=65$ $\mathrm{min} / \mathrm{sem})$.

O domínio AF doméstica inclui as atividades que as idosas executam numa semana normal dentro e ao redor da sua habitação (casa ou apartamento). Das idosas que fazem atividades ao redor da habitação (pátio, quintal ou jardim), 36.9\% fazem-no com intensidade moderada $(\overline{\mathrm{X}}=107 \mathrm{~min} / \mathrm{sem})$ e apenas $8.6 \%(\overline{\mathrm{X}}=125 \mathrm{~min} / \mathrm{sem})$ com intensidade vigorosa. Dentro da habitação, $61.1 \%$ realizam atividades domésticas com intensidade moderada, em média de $230 \mathrm{~min} / \mathrm{sem}$.

$\mathrm{O}$ domínio $\mathrm{AF}$ de lazer refere-se às atividades físicas de recreação, desporto, exercício ou lazer que as idosas executam numa semana normal. Assim, estas apresentam como atividade física a caminhada, sendo que $30.3 \%$ o fazem com intensidade moderada $(\bar{X}=239 \mathrm{~min} / \mathrm{sem}), 14.6 \%$ com leve $(\overline{\mathrm{X}}=170$ $\mathrm{min} / \mathrm{sem})$ e $1.0 \%$, com vigorosa $(\overline{\mathrm{X}}=180 \mathrm{~min} / \mathrm{sem})$. As atividades físicas de lazer de intensidade vigorosa não são realizadas pelas idosas, mas as de intensidade moderada são executadas por $52.5 \%$ destas, em média de $112 \mathrm{~min} / \mathrm{sem}$.

O domínio tempo sentado relaciona-se com o tempo que as idosas permanecem sentadas em diferentes locais, durante um dia da semana normal e um dia do fim-de-semana. Também incluiu-se neste domínio o tempo gasto sentado durante uma semana normal no transporte em ônibus, carro e outros veículos. Todas as idosas do estudo despendem tempo na posição sentada durante um dia da semana e um dia no fim-de-semana, destacando-se com maior tempo no fim-de-semana ( $\bar{X}=472 \mathrm{~min} /$ dia $)$ em relação à semana $(\bar{X}=421 \mathrm{~min} / \mathrm{dia})$. Destas, $77.3 \%$ gastam, em média, $176 \mathrm{~min} / \mathrm{sem}$ de tempo sentadas durante o transporte em veículos, para se deslocar de um lugar para o outro.

Por outro lado, as mulheres idosas deste estudo apresentam um período de tempo em minutos por semana ( $\mathrm{min} / \mathrm{sem})$ de atividades moderadas e vigorosas nas diferentes formas de atividades físicas, conforme a Figura 1.

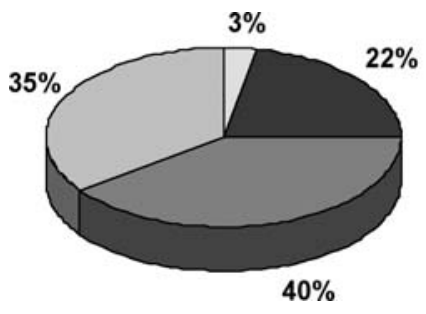

\begin{tabular}{l}
\hline$\square$ AF Trabalho \\
$\square$ AF Transporte \\
$\square$ AF Doméstica \\
$\square$ AF Lazer
\end{tabular}

Figura 1. Contribuição percentual das diferentes formas de atividade física[AF] moderadas e vigorosas em minutos por semana [períodos mínimos de 10 minutos contínuos].

Os resultados indicam que o período de tempo (min/sem) de atividades moderadas e vigorosas, por, pelo menos, 10 minutos contínuos, destaca-se nas atividades físicas domésticas e de lazer.

Para a interpretação do nível de atividade física das idosas através do IPAQ, a amostra foi dividida em dois níveis de atividade física (menos e mais ativas), sendo que $33.8 \%$ das idosas foram consideradas menos ativas e $66.2 \%$ mais ativas (Tabela 2 ).

Na Tabela 2, apresenta-se a relação entre o nível de atividade física (menos e mais ativo) com as características sócio-demográficas das idosas. 
Tabela 2. Número [ $\mathrm{n}$ ], percentagem [\%] e teste do Qui-Quadrado [ $\chi^{2}$ ] entre o nível de atividade física (menos e mais ativo] e as características sócio-demográficas da amostra. Florianópolis, SC, Brasil, 2001.

\begin{tabular}{|c|c|c|c|c|c|c|}
\hline \multirow{3}{*}{$\begin{array}{l}\text { Características } \\
\text { Sócio-Demográficas }\end{array}$} & \multicolumn{4}{|c|}{ Nível de Atividade Física } & \multirow{2}{*}{\multicolumn{2}{|c|}{$x^{2}$}} \\
\hline & \multicolumn{2}{|c|}{ Menos Ativo } & \multicolumn{2}{|c|}{ Mais Ativo } & & \\
\hline & $n$ & $\%$ & n & $\%$ & valor & $p$ \\
\hline Estrato Etário & & & & & \multirow{5}{*}{23.4} & \multirow{5}{*}{$<0.001$} \\
\hline 65.69 & 7 & 12.3 & 50 & $87.7^{\circ}$ & & \\
\hline $70 \cdot 74$ & 20 & 33.9 & 39 & 66.1 & & \\
\hline 75.79 & 19 & 40.4 & 28 & 59.6 & & \\
\hline$>80$ & 21 & $60.0^{\circ}$ & 14 & 40.0 & & \\
\hline \multicolumn{5}{|l|}{ Estado Civil } & \multirow{5}{*}{7.9} & \multirow{5}{*}{$<0.050$} \\
\hline Solteira & 4 & 36.4 & $?$ & 63.6 & & \\
\hline Casada & 11 & 19.6 & 45 & $80.4^{a}$ & & \\
\hline Divorciada/Separada & 3 & 27.3 & 8 & 72.7 & & \\
\hline Viúva & 49 & $40.8^{\circ}$ & 71 & 59.2 & & \\
\hline \multicolumn{5}{|l|}{ Origem Étnica } & \multirow{5}{*}{10.3} & \multirow{5}{*}{$<0.050$} \\
\hline Brasileira & 36 & $46.2^{\circ}$ & 42 & 53.8 & & \\
\hline Miscigenada & 14 & 30.4 & 32 & 69.6 & & \\
\hline Européia & 14 & 21.2 & 52 & $78.8^{\circ}$ & & \\
\hline Desconhecida & 3 & 37.5 & 5 & 62.5 & & \\
\hline \multicolumn{5}{|l|}{ Escolaridade } & \multirow{5}{*}{13.2} & \multirow{5}{*}{$<0.010$} \\
\hline Sem instrução & 18 & $60.0^{\circ}$ & 12 & 40.0 & & \\
\hline 1 a 3 anos & 42 & 30.4 & 96 & 69.6 & & \\
\hline 4 a $?$ anos & 5 & 35.7 & 9 & 64.3 & & \\
\hline$>8$ anos & 2 & 12.5 & 14 & 87.5 & & \\
\hline \multicolumn{5}{|l|}{ Religião } & \multirow{5}{*}{3.3} & \multirow{5}{*}{0.349} \\
\hline Católica & 58 & 33.1 & 117 & 66.9 & & \\
\hline Evangélica & $?$ & 53.9 & 6 & 46.2 & & \\
\hline Espírita & 1 & $16 . ?$ & 5 & 83.3 & & \\
\hline Outras & 1 & 25.0 & 3 & 75.0 & & \\
\hline \multicolumn{5}{|l|}{ Trabalho } & \multirow{3}{*}{0.1} & \multirow{3}{*}{0.730} \\
\hline Sim & 9 & 31.0 & 20 & 69.0 & & \\
\hline Não & 58 & 34.3 & 111 & 65.7 & & \\
\hline \multicolumn{5}{|l|}{ Trabalho Voluntário } & \multirow{3}{*}{4.5} & \multirow{3}{*}{$<0.050$} \\
\hline Sim & 3 & 13.6 & 19 & $86.4^{\circ}$ & & \\
\hline Não & 64 & $36.4^{\circ}$ & 112 & 63.6 & & \\
\hline \multicolumn{5}{|l|}{ Classe Econômica } & & \\
\hline B & $?$ & 50.0 & $?$ & 50.0 & & \\
\hline C & 17 & 29.8 & 40 & 70.2 & 7.5 & $0.05 ?$ \\
\hline$D$ & 33 & 30.0 & 77 & 20.0 & & \\
\hline E & 10 & $58.8^{a}$ & $?$ & 41.2 & & \\
\hline Total (n e \%) & $6 ?$ & 33.8 & 131 & 66.2 & & \\
\hline
\end{tabular}

a Valor do resíduo ajustado >[2] 
Entre o nível de AF e as características sócio-demográficas há uma diferença estatisticamente significativa para o estrato etário $(\mathrm{p}<0.001)$, estado civil $(\mathrm{p}<0.050)$, origem étnica $(\mathrm{p}<0.050)$, escolaridade $(\mathrm{p}<0.010)$ e trabalho voluntário $(\mathrm{p}<0.050)$. Verifica-se que as idosas menos ativas estão mais relacionadas com o estrato etário de 80 anos ou mais de idade $(60.0 \%)$, estado civil "viúva" (40.8\%), origem étnica "brasileira" $(46.2 \%)$, sem instrução escolar (60.0\%) e não fazem trabalho voluntário (36.4\%), sendo que as idosas mais ativas estão mais relacionadas com o estrato etário de 65 a 69 anos (87.7\%), estado civil "casada" (80.4\%), ori- gem étnica "européia" (78.8\%) e realizam trabalho voluntário $(86.4 \%)$.

Não há uma diferença estatisticamente significativa entre o nível de AF e as seguintes características sócio-demográficas: religião $(\mathrm{p}=0.349)$, trabalho $(\mathrm{p}=0.730)$ e classe econômica $(\mathrm{p}=0.057)$. Apesar disso, observa-se que as idosas menos ativas parecem relacionar-se mais com a classe econômica "E" (58.8\%), que é a classe mais baixa em termos de poder de consumo e de rendimento mensal familiar. Outro aspecto importante é a relação entre o nível de atividade física e as condições de saúde da amostra (Tabela 3).

Tabela 3. Número ( $n$ ), percentagem [\%] e teste do Qui-Quadrado $\left[\chi^{2}\right]$ entre o nível de atividade física (menos e mais ativo] e as condições de saúde da amostra. Florianópolis, SC, Brasil.

\begin{tabular}{|c|c|c|c|c|c|c|}
\hline \multirow{3}{*}{ Condições de Saúde } & \multicolumn{4}{|c|}{ Nível de Atividade Física } & \multirow{2}{*}{\multicolumn{2}{|c|}{$x^{2}$}} \\
\hline & \multicolumn{2}{|c|}{ Menos Ativo } & \multicolumn{2}{|c|}{ Mais Ativo } & & \\
\hline & n & $\%$ & $\mathrm{n}$ & $\%$ & valor & p \\
\hline \multicolumn{7}{|l|}{ Doenças } \\
\hline Sim & 65 & $38.2^{\circ}$ & 105 & 61.8 & 10.4 & $<0.010$ \\
\hline Não & 2 & 7.1 & 26 & $92.9^{a}$ & & \\
\hline \multicolumn{7}{|c|}{ Estado de saúde dificulta AF } \\
\hline Sim & 25 & $45.5^{\circ}$ & 30 & 54.5 & 4.6 & $<0.050$ \\
\hline Nảo & 42 & 29.4 & 101 & $20.6^{a}$ & & \\
\hline \multicolumn{7}{|l|}{ Percepção de saúde } \\
\hline Positiva & 56 & 32.2 & 118 & 67.8 & 1.8 & 0.185 \\
\hline Negativa & 11 & 45.8 & 13 & 54.2 & & \\
\hline \multicolumn{7}{|l|}{ Satisfação com a saúde } \\
\hline Positiva & 42 & 32.1 & 89 & 67.9 & 0.6 & 0.460 \\
\hline Negativa & 25 & 37.3 & 42 & 62.7 & & \\
\hline Total (n e \%) & 67 & 33.8 & 131 & 66.2 & & \\
\hline
\end{tabular}

${ }^{a}$ Valor do resíduo ajustado $>$ [2]

Entre o nível de AF e as condições de saúde há uma diferença estatisticamente significativa no que se refere às doenças $(\mathrm{p}<0.010)$ e ao fato de o estado de saúde atual dificultar ou não a prática de atividade física $(\mathrm{p}<0.050)$. As idosas menos ativas estão mais relacionadas com a presença de doença (38.2\%) e com o estado de saúde, dificultando a prática de AF (45.5\%), enquanto que as idosas mais ativas o estão com a ausência de doenças (92.9\%) e com o estado de saúde atual, não dificultando a prática de $\mathrm{AF}$ $(70.6 \%)$. Não há uma diferença estatisticamente significativa entre a percepção de saúde $(\mathrm{p}=0.185)$ e a satisfação com a saúde $(\mathrm{p}=0.460)$.

\section{DISCUSSÃO}

As atividades físicas diárias são importantes para que os idosos permaneçam com uma melhor aptidão física, pois requerem um nível mínimo de força muscular, flexibilidade, coordenação e equilíbrio $(2,9,13)$ e, com isto, mantenham sua capacidade funcional.

A atividade física no trabalho, no nosso estudo, não representa uma influência significativa no dispêndio energético requisitado para uma vida ativa das idosas, visto que a maioria delas não trabalha.

A atividade física no transporte relaciona-se com o deslocamento de um lugar para o outro. Neste estu- 
do, é realizada principalmente através da caminhada, numa intensidade moderada, surgindo associada às compras, à visita a parentes e vizinhos e à ida ao Grupo de Convivência para Idosos. Isto parece constituir uma ação utilitária importante, já que caminhar é conveniente e pode incluir rotinas ocupacionais e domésticas, onde nenhuma habilidade específica ou equipamento são necessários. A aptidão física adquirida pela caminhada parece ser valiosa para os idosos, pois a força dos membros inferiores auxilia a minimizar os efeitos da imobilidade e, conseqüentemente, a manter a independência. A caminhada é auto-regulável em termos de intensidade, duração e freqüência e tem um baixo impacto, sendo uma excelente opção para aumentar o nível de atividade física das idosas $(25,15)$. Além disso, níveis moderados de $\mathrm{AF}$, como a caminhada, são associados significativamente à redução do risco de fratura do quadril em mulheres após a menopausa (12).

A maioria das idosas, no nosso estudo, realiza atividades domésticas dentro e ao redor da habitação, com uma intensidade moderada, mantendo-se, desta forma, ativas no seu dia-a-dia. As atividades físicas mais comuns, realizadas pelas idosas, estão relacionadas com as tarefas domésticas, como cozinhar, cuidar do jardim e limpeza da moradia, tarefas estas importantes para a análise do nível de atividade física das idosas (34). Ainsworth (3) inclui as mulheres num grupo de pessoas muito ativas durante as suas vidas, devido às atividades domésticas. Estima-se que estas despendem, em média, 3.9 horas por dia em trabalhos domésticos e em tarefas de cuidados com a família, sendo que o tempo de atividade física total é de $421 \mathrm{MET} / \mathrm{min} /$ dia.

Em estudo de cunho epidemiológico com idosos residentes em Florianópolis, as mulheres realizavam atividades domésticas moderadas ou vigorosas por um tempo médio de 227.5 minutos por semana, demonstrando serem mais ativas do que os homens nesta tarefa (7).

No nosso estudo, as atividades físicas de recreação, desporto, exercício ou lazer moderadas ou vigorosas são praticadas por $35 \%$ das mulheres idosas, normalmente, realizadas através da caminhada, como exercício físico com intensidade moderada e das atividades de ginástica e natação, também com essa intensidade. Em estudo sobre o perfil do idoso de
Florianópolis verificou-se que $39.7 \%$ dos idosos realizam atividades físicas de lazer (7).

Comparando-se com estudo realizado com idosos de ambos os sexos que residem em Florianópolis, observa-se que $60.3 \%$ não realizam nenhuma atividade de lazer, mas os que praticam (39.7\%) apresentam um tempo médio de 272.5 minutos por semana (7), valor este superior encontrado em nossa pesquisa. Acredita-se que isto se deve ao fato da presença do sexo masculino neste estudo, onde os homens são mais ativos do que as mulheres em $30.4 \%$ nestas atividades. As atividades físicas mais praticadas são a caminhada, seguida pela ginástica.

Num estudo sobre a população portuguesa com 65 anos ou mais de idade, Faria (11) observou que a taxa percentual de idosos que não praticam qualquer atividade física desportiva (72\%) atinge a sua maior expressão. Dos $28 \%$ que praticam atividades de forma regular, $9 \%$ praticam natação e $7 \%$ a caminhada, destacando-se ambas de forma significativa, em termos percentuais, das restantes atividades mais praticadas. Este autor também verificou um declínio das atividades físicas desportivas coletivas face às individuais, destacando as AFs não organizadas, como caminhadas e jogging, entre outras, que levam o idoso a tornar-se mais ativo.

Os resultados obtidos vão ao encontro do que nos dizem Yusuf et al. (41), quando referem precisamente que a caminhada, a ginástica e a natação constituem as principais atividades físicas das mulheres idosas. Também, a caminhada e a ginástica são as formas de exercício mais populares em idosas filandesas, onde $2 / 3$ desta população caminha regularmente e $1 / 3$ faz ginástica em casa, apesar de $o$ envolvimento nessas atividades começar a diminuir nas idosas com 80 anos ou mais de idade (15). A reduzida participação das idosas em desportos pode ser atribuída aos estereótipos relacionados com o gênero, à discriminação da idade, à falta de suporte/apoio social (família, amigos e colegas), de hábito durante os períodos de vida, de ambiente físico apropriado, de motivação ou "força de vontade", de recursos financeiros, de conhecimento e informação sobre programas desportivos, ao estado de saúde, ao medo de quedas e das suas conseqüências e à falta de uma política de promoção de saúde, através do desporto, entre outros motivos $(15,23,36,40)$. 
Ao estudar idosos brasileiros atletas de natação e de atletismo no Rio de Janeiro, Santiago (31) evidenciou a predominância do sexo masculino entre estes. Com efeito, os homens praticam estes desportos desde a juventude. Já as mulheres, em menor número, começaram a praticá-los numa idade avançada, por incentivo da família ou de amigos.

Outro aspecto que influencia o nível de atividade física habitual das idosas é o tempo que passam diariamente na posição sentada. Neste aspecto, observa-se, no nosso estudo, que todas as idosas despendem muito tempo nesta posição, principalmente no fim-de-semana. Também a maioria delas passa muito tempo sentada em meios de transporte durante uma semana normal. Além disso, ficam, muitas vezes, sentadas, assistindo televisão.

Em estudo com idosos de ambos os sexos que residem em Florianópolis, observou-se que os idosos menos ativos ficam de 10 a 15 horas por dia sentados assistindo televisão, ouvindo rádio e recebendo visitas e os mais ativos de 5 a 9 horas (7).

No Brasil, assistir televisão é o maior componente na ocupação do tempo livre das pessoas (17). De fato, esta atividade constitui uma das principais ocupações do tempo livre, com um baixo dispêndio energético, não exigindo nem desenvolvendo nenhuma capacidade física $(4,40)$, sendo que a falta de atividade física e a quantidade de horas passadas assistindo televisão foram associadas significativamente aos marcadores bioquímicos de obesidade e ao risco de doenças cardiovasculares (14).

Ao estudar mulheres idosas, Souza (35) verificou que as principais atividades do dia-a-dia, para a maioria delas, consistiam em assistir televisão, ouvir rádio, receber e fazer visitas. Também Anderson et al. (5) verificaram que as atividades mais comuns, realizadas no lar pelos idosos, eram ver televisão, ouvir rádio, ler e costurar. Estas atividades são predominantemente de intensidade leve.

As recomendações atuais para se conseguir benefícios no que respeita à saúde através da atividade física (no trabalho, nas atividades domésticas, no transporte, no lazer, recreação e desporto) são a prática desta com uma intensidade moderada, durante, pelo menos, 30 minutos por dia, na maior parte dos dias da semana, de preferência todos os dias, de forma contínua ou acumulada (28).
Assim, constata-se que grande parte das idosas (66.2\%) que participam nos Grupos de Convivência para Idosos em Florianópolis, SC, no Brasil, pertence ao nível de atividade física mais ativo, em destaque nas atividades físicas relacionadas as tarefas domésticas $(40 \%)$ e de lazer (35\%). Em estudo realizado no município de Marechal Cândido Rondon, no estado do Paraná, no Brasil, com 320 mulheres idosas (com 60 ou mais anos de idade) que participavam de Grupo de Convivência, observou-se que $76.5 \%$ são ativas fisicamente (10).

Neste contexto, observa-se que as idosas que participam de Grupos de Convivência são mais ativas na sua vida diária, tanto nas atividades domésticas, como de lazer (caminhada, ginástica, natação e atividades recreativas). Nos Grupos de Convivência são realizadas atividades diversas, como de lazer, culturais, intelectuais, físicas, manuais, artísticas e de convívio grupal que podem favorecer uma vida ativa as idosas.

Em relação ao nível de atividade física e às características sócio-demográficas das mulheres idosas, no nosso estudo, observou-se que as idosas menos ativas são mais velhas, "viúvas", de origem étnica "brasileira", possuem um menor nível de escolaridade e não fazem trabalho voluntário.

Em relação ao nível de AF e à escolaridade das mulheres idosas, no nosso estudo, observou-se que, quanto mais baixo o nível de AF, menor é o nível educacional das mesmas. Resultados de diferentes estudos corroboram estes dados, ao confirmarem que a prática de atividade física no tempo livre se encontra relacionada com a idade e o nível educacional, sendo que a população com um nível educacional mais alto tem uma correlação mais forte com atividades de intensidade vigorosa e moderada, relativamente aos restantes níveis educacionais analisados (8); idosos com maior nível de escolaridade são mais ativos (7); a prevalência da inatividade física diminui com o aumento dos níveis de educação e rendimentos $(29,38,41)$, além de se sugerir que pode haver diferenças entre os aspectos étnicos (38). $\mathrm{Na}$ sociedade brasileira, verifica-se que o nível de escolaridade é outro fator que contribui para o reduzido número de idosos praticantes de desportos, principalmente entre as mulheres, pois a maioria dos idosos apresenta de um a três anos de estudos e não 
teve, no período escolar, a oportunidade de vivenciar o desporto nas aulas de Educação Física. Portanto, este não fazia parte da sua educação e do seu cotidiano (22).

A maioria das idosas do nosso estudo não desenvolve um trabalho remunerado e voluntário, mas observa-se que as que se envolvem em trabalhos voluntários (principalmente com o atendimento ao Grupo de Convivência e com atividades relacionadas à igreja) são mais ativas. Neste atendimento, as idosas têm responsabilidades de organização, ensino e coordenação de atividades, que requerem um dinamismo e uma movimentação física constantes.

A pequena adesão ao trabalho voluntário por parte das idosas também é referida no estudo de Anderson et al. (5), onde apenas $28 \%$ dos idosos desenvolviam atividades assistenciais ligadas a obras religiosas e/ou beneficentes, apesar de um número expressivo de idosos (51.6\%), revelar interesse em desenvolver um trabalho comunitário, o que demonstra a necessidade de ampliar e diversificar espaços de atuação neste campo.

A maioria das idosas deste estudo são "viúvas", podendo constatar-se que estas são menos ativas do que as "casadas". Isto pode ser explicado pelo fato de as idosas casadas terem de acompanhar os maridos nas suas atividades. Rowland (29) também refere que os sujeitos que possuem o suporte dos amigos e dos cônjuges têm uma maior probabilidade de serem fisicamente ativos.

Em relação ao nível de atividade física e às condições de saúde das idosas observa-se, no nosso estudo, que as idosas menos ativas apresentam doenças e que os seus estados de saúde dificultam a prática de AF, enquanto que as mais ativas não têm doenças e os seus estados de saúde não dificultam a prática de AF. A literatura é cada vez mais consensual quanto à possível relação entre estilos de vida menos ativos e o incremento de determinadas doenças características das sociedades industriais $(24,30)$. Com efeito, os indivíduos que vivem com inaptidões e doenças crônicas são os segmentos menos ativos da população $(18,38)$. Pelo contrário, os sujeitos mais saudáveis são também mais ativos, mesmo que possuam alguma doença associada às limitações funcionais (37). $\mathrm{O}$ estilo de vida tem um papel fundamental na promoção de saúde e na qualidade de vida durante o processo de envelhecimento. $\mathrm{O}$ fator idade não é, por si só, um obstáculo para o exercício físico. Diferentes estudos têm demonstrado que o declínio físico e funcional associado ao envelhecimento pode, mesmo em sujeitos com idade avançada, ser revertido através do exercício físico $(13,20,27,33)$. O exercício físico pode contribuir com mudanças positivas e aumentar a capacidade física do idoso. Deste modo, no nosso estudo, verifica-se que a maioria das idosas que participa em Grupos de Convivência para Idosos em Florianópolis é ativa. Apesar disso, deve intervir-se nesta realidade, incentivando as idosas que são menos ativas a tornarem-se mais ativas, e as mais ativas a manterem-se ou a aumentarem este nível.

\section{CONCLUSÃO}

Em relação às atividades físicas com intensidade leve, moderada e vigorosa nos domínios do IPAQ, observou-se que: a AF no trabalho não representa uma influência significativa no dispêndio energético das idosas, visto que a maioria delas não trabalha; no transporte, ela é realizada principalmente através da caminhada; nas atividades domésticas, ocorre dentro e ao redor da habitação, com uma intensidade moderada; as AFs de recreação, desporto, exercício ou lazer, que as idosas executam, são, normalmente, realizadas através da caminhada, como exercício físico, e das atividades de ginástica e natação, com intensidade moderada. Também as idosas despendem muito tempo na posição sentada, principalmente nos fins-de-semana e em meios de transporte. Em relação às AFs com intensidades moderadas e vigorosas, por, pelo menos, 10 minutos contínuos por semana, destacam-se as atividades domésticas e de lazer e, depois, as atividades de transporte e de trabalho. Assim, grande parte das idosas que participam nos Grupos de Convivência para Idosos pertence ao nível de AF mais ativo.

Também o nível de AF está relacionado com as características sócio-demográficas e as condições de saúde, sendo que as idosas mais ativas são mais jovens, "casadas", de origem étnica "européia", possuem um melhor nível de escolaridade, realizam trabalho voluntário, não têm doenças e o seu estado de saúde não dificulta a prática de AF.

Diante destas conclusões, constata-se que a AF tem um papel importante no estilo de vida ativo e saudá- 
vel das idosas. Assim, torna-se necessário intervir nesta realidade, através de divulgação dos programas de atividade física, de informações na mídia, em folhetos explicativos e palestras sobre a importância da prática de atividade física, de recursos financeiros para melhorar as condições de vida dos idosos, da qualificação de profissionais para atuar nesta faixa etária, para que as idosas menos ativas se tornem ativas e as mais ativas se mantenham ou aumentem o seu nível de AF.

\section{Agradecimentos}

Os dados deste artigo são referentes à tese de doutoramento intitulada "Atividade física e qualidade de vida de mulheres idosas" da autora Giovana Zarpellon Mazo, que realizou esta na Faculdade de Ciências do Desporto e de Educação Física, Universidade do Porto, Porto, Portugal, em 2003. A pesquisa foi financiada pela CAPES

\section{CORRESPONDÊNCIA}

\section{Giovana Zarpellon Mazo}

Rua Procópio Manoel Pires no 153 apt $^{\mathrm{o}} 105$

Bairro: Trindade

88036-090 Florianópolis, SC,

Brasil

d2gzm@udesc.br

\section{REFERÊNCIAS}

1. ACSM - American College of Sports Medicine (2000). ACSM's guidelines for exercise testing and prescription. $6^{\mathrm{a}}$ ed. Philadelphia: Lippincott Willians \& Wikins.

2. Adams, K.; O'Shea, P.; O'Shea, K.L. (1999). Aging: its effects on strength, power, flexibility, and bone density. Natl. Strength Cond. Assoc. J. 21, 65-77.

3. Ainsworth, B. (2000). Issues in the assessment of physical activity in woman. Res. Q. Exerc. Spor. 71 (2)37-42.

4. Allsen, P.E.; Harrison, J.H.; e Vance, B. (2001). Exercício e qualidade de vida: uma abordagem personalizada. São Paulo: Manole.

5. Anderson, M.I.P.; Assis, M.; Pacheco, L.C.; Menezes, I.S.; Silva, E.A.; Duarte, T.; Storino, F.; Motta, L. (1998). Saúde e qualidade de vida na terceira idade. Equipe de Saúde da UnATI/Núcleo de Atenção ao Idoso - NAI. [On-line]: www.unati.uerj.br.

6. ANEP - Associação Nacional de Empresas de Pesquisa (1997). Critério de Classificação Econômica - Brasil. [On-line]: www.anep.org.br/mural/anep.

7. Benedetti, T.B (2004). Atividade física: uma perspectiva de promoção de saúde do idoso no município de Florianópolis.

Universidade Federal de Santa Catarina, Centro de Ciência de Saúde, Doutorado em Enfermagem (Tese de Doutorado)

8. Boffetta, P.; Barone, J.; Wynder, E. (1990). Leisure time physical activity in a Hospital-Based population. J. Clin. Epidem. 43 (6)569-577.

9. Brill, P.; Macera, C.; Davis, D.; Blair, S.; Gordon, N. (2000). Muscular strength and physical function. Med. Sci. Sports Exerc. 32, 412-416.

10. Conte, E.M.T. (2004). Indicadores de qualidade de vida em mulheres idosas. Florianópolis, Universidade Federal de Santa Catarina, Centro de Desportos (Dissertação de Mestrado)

11. Faria, J.C.G. (2001). Caracterização da actividade fisica habitual da população portuguesa. Lisboa, Universidade Técnica de Lisboa, Faculdade de Motricidade Humana (Dissertação de Mestrado).

12. Feskanich, D.; Willett, W.; Colditz, G. (2002). Walking and leisure-time activity and risk of hip fracture in postmenopausal women. JAMA 288 (18) 2300-2306.

13. Fiatarone, M.; O'Neill, E.; Doyle, N.; Clements, K.; Roberts, S.; Kehayias, J.; Lipsitz, L.; Evans, W. (1994). Exercise training and supplementation for physical frailty in very elderly people. N. Engl. J. Med. 330, 1769-1775.

14. Fung, T.T.; Frank, B.H.; Ji Hu; Nain-Feng Chu; Spielgelman, D.; Tofleer, G.H.; Willett, W.C.; Rimm, E.B. (2000). Leisure-time physical activity, television watching, and plasma biomarkers of obesity and cardiovascular disease risk. Am. J. Epidem. 152, 1171-1178.

15. Heikkinen, E.; Heikkinen, RL.; Kauppinen, M.; Laukkanen, P.; Ruoppila, I.; Suutama, T. (1990). General issues regarding ageing and technology. [On-line]: www.stakes.fi/include/index.html.

16. Hallal, P.C.; Victora, C.G.; Wells, J.C.K.; Lima, R.C. (2003) Physical inactivity: prevalence and associated variables in Brazilian adults. Med.Sci.Sports Exerc. 35 (11)1894-1900.

17. IBGE - Instituto Brasileiro de Geografia e Estatística (1998). Pesquisa sobre Padrões de Vida. Brasília. [On-line]: www.ibge.gov.br/imprensa/noticias/ppv11.html.

18. King, A.C.; Oman, R.E.M.; Brassington, G.S.; Bliwise, 
D.L.; Haskell, W.L.. (1998). Moderate-intensity exercise and self-related quality of sleep in older adults. JAMA v. 277: 1, 32-37.

19. Lee, I.; Paffenbarger R.S. (2000). Associations of light, moderate, and vigorous intensity physical activity with longevity. Am. J. Epidem. 151, 293-299.

20. Lee, I.; Rexrode, K.; Cook, N.; Manson, J.; Buring, J. (2001). Physical activity and coronary heart disease in women. Is no pain, no gain passé?. JAMA 285, 1447-1454.

21. Marshall, A.; Bauman, A. (2001). The International Physical Activity Questionnaire: Summary Report of the reliability \& validity studies. Summary, march.

22. Mazo, G.Z.; Lopes, M.A.; Benedetti, T.B. (2001). Atividade física e o idoso: concepção gerontológica. Porto Alegre: Sulina.

23. McArdle, W.D.; Katch, F.I.; Katch V.L. (1998). Fisiologia do Exercício, Energia, Nutrição e Desempenho Humano. $4^{\text {a }}$ ed. Rio de Janeiro: Guanabara Koogan.

24. Montoye, H.J.; Kemper, H.C.G.; Saris, W.H.M.; Washburn, R.A. (1996). Measuring Physical Activity and Energy Expenditure. Champaign, Illinois: Human Kinetics.

25. Morris, J.N.; Hardman, A. (1997). Walking to Health. Sports Med. 23 (5)306-332.

26. OPAS - Organização Pan-Americana de Saúde (1998). La salud em las Américas (V.1). Washington: OPAS.

27. Paffenbarger, R.S..; Lee, I. (1996). Physical activity and fitness for health and longevity. Res. Q. Exerc. Sport 67, 11-28.

28. Pate, R.R.; Pratt M.; Blair, S.N.; Haskell, W.L.; Macera, C.A.; Bouchard, C.; Buchner, D.; Ettinger, W.; Heath, G.W.; King, A.C.; Kriska, A.; Leon, A.S.; Marcus, B.H.; Morris, J.; Paffenbarger, R.S.; Patrick, K.; Pollock, M.L.; Rippe, J.M.; Sallis, J.; Wilmore, L.L.I (1995). Physical activity and public health: A recommendation from the Centers for Disease Control and Prevention and the American College of Sports Medicine. JAMA 273, 402-407.

29. Rowland, T. (1998). The Biological Basis of Physical Activity. Med. Sci. Sports Exerc. 30 (3)392-399.

30. Sallis, L.F.; Hovell, M.F; Hofsletter, C.R.; Faucher P.; Elder, P.; Blanchard L.; Caspersen, C.L.; Powell K.E.; Christenson, G.M. (1989). A multivariate study of determinants of vigorous exercise in a community sample. Prev. Med. 18, 20-34.

31. Santiago, L. V. (1999). Os Valores Orientadores das Práticas Desportivas em Grupos Emergentes da Terceira Idade: um estudo sobre suas construçôes simbólicas. Porto, Faculdade de Ciências de Desporto e de Educação Física da Universidade de Porto (Tese de Doutorado).

32. Shephard, R.J. (1995). Physical activity, fitness and health: the current consensus. QUEST 47, 288-303.

33. Shephard, R.J. (2002). Gender, Physical Activity and Aging. Boca Raton, Florida: CRC Press.

34. Sihvonen, S.; Rantanen, T.; Heikkinen, E. (1998). Physical activity and survival in elderly people: a five-year follow-up study. J. Aging Phys. Act. 6, 133-140.

35. Souza, A.I. (2001). A visão das mulheres idosas em relação à atenção à saúde e o apoio social em uma localidade de baixa renda do Rio de Janeiro. Rio de Janeiro, Fundação Oswaldo Cruz, Saúde da Mulher e da Criança (Tese de Doutorado).

36. Spirduso, W.W.; Gilliam-MacRae, P. (1991). Physical Activity and Quality of Life in the Frail Elderly. In: Birren, J.E.; Lubben, J.; Rowe, J.C.; Deutchman, D.E. (Eds.). The concept and measurement of quality of life in the frail elderly. San Diego, California: Academic Press, 226-255.

37. Stewart, A.L.; Hays, R.D.; Wells, K.; Rogers, W.H.;
Greenfield, S. (1994). Long-term functioning and wellbeing outcomes associated with physical activity and exercise in-patients with chronic conditions in the Medical Outcomes Study. J. Clin. Epidem. 47, 719-730.

38. USDHHS - U.S. Department of Health and Human Services (1996). Physical Activity and Health: a Report of the Surgeon General. Atlanta: U.S. Departament of Health and Human Servives, Centers for Disease Control and Prevention, National Center for Chronic Disease Prevention and Health Promotion.

39. WHO - World Health Organization (1997). Declaración de Yakarta. In: P.M. Buss (Ed.) Promoção da Saúde e Saúde Pública. Rio de Janeiro: ENSP, 174-178.

40. Wilcox, S.; Tudor-Locke, C.E.; Ainsworth, B.E. (2002). Physical Activity Patterns, Assessment, and Motivation in Older Adults. In: R.J. Shephard (Ed.) Gender, Physical Activity, and Aging. Florida: CRC Press LLC, 13-39.

41. Yusuf, H.; Croft, J.; Giles, W.; Anda, R.; Casper, M.; Caspersen, C.; Jones, D. (1996). Leisure-time physical activity among older adults. Arch. Internal Med. 156 (12) 1321-1326. 